\title{
Molecular Mechanisms for Alcoholic Hepatitis Based on Analysis of Gene Expression Profile
}

\author{
Minghui Liu ${ }^{1}$; Yuchang Dou ${ }^{1,}$; Ran Sun ${ }^{2}$; Yonggui Zhang ${ }^{3}$; Yansong Liu ${ }^{1}$ \\ ${ }^{1}$ Department of TCM, China-Japan Union Hospital of Jilin University, Changchun, China \\ ${ }^{2}$ Science Research Center, China-Japan Union Hospital of Jilin University, Changchun, China \\ 3 Department of Gastroenterology, China-Japan Union Hospital of Jilin University, Changchun, China \\ *Corresponding Author: Yuchang Dou, Department of TCM, China-Japan Union Hospital of jilin University, Changchun, China. Tel: +86-43184995840, Fax:+86-43184995840, \\ E-mail:yuchangdouycd@163.com
}

Received: January 25, 2015; Revised: March 4, 2015; Accepted: March 29, 2015

\begin{abstract}
Background: Alcoholic hepatitis (AH) is an acute manifestation of alcoholic liver disease with high mortality rates.
Objectives: Our aim was to study the molecular mechanisms of AH.

Materials and Methods: The differentially expressed genes (DEGs) in liver between AH and control cases were identified by analyzing the GSE28619 microarray data using t-test. The Kyoto Encyclopedia of Genes and Genomes (KEGG) pathways and Gene Ontology (GO) enrichment analyses were performed usingDAVID online tool. The protein-protein interaction(PPI) network was constructed using Search Tool for the Retrieval of Interacting Genes (STRING) and the subnetwork was identified by BioNet. Both PPI network and subnetwork were visualized using the Cytoscape software.

Results: Total 908 DEGs (551 up- and 357 down-regulated DEGs) were obtained. The up-regulated DEGs were significantly enriched in 15 pathways and 112 GO biological processes. The down-regulated DEGs were significantly enriched in 22 pathways and 84 GO biological processes. The PPI network with 608 nodes and 2878 interactions was constructed and the subnetwork with 53 nodes and 131 interactions was also identified. The hub DEGs (TSPO, PPIB, NME1 and NME2) were extracted in this subnetwork.

Conclusions: TSPO might contribute to the liver damage and AH progression induced by mitochondrial dysfunction through oxidative stress of liver. TSPO interacted with PPIB might play important roles in liver damage in AH. The interaction between NME1 and NME2 might contribute to the transformation from $\mathrm{AH}$ to hepatocellular carcinoma.
\end{abstract}

Keywords: Protein-Protein Interaction Map; Alcoholic Hepatitis; Disease

\section{Background}

Alcoholic hepatitis (AH) is an acute manifestation of alcoholic liver disease, which has a poor short-term prognosis and high mortality rates (40-50\%) in severe cases (1-3). It is often severe and characterized by tender hepatomegaly, hepatocellular damage, steatosis and pericellular fibrosis $(2,3)$. Patients with severe AH generally present with fever, anorexia, fatigue, jaundice, and ascites (3). Currently, the main therapy for $\mathrm{AH}$ is pharmacological therapy mainly including pentoxifylline or corticosteroids, but the treatment outcomes were poor $(4,5)$. Consequently, studying the molecular mechanisms of AH may offer a new hope on discovery of new targeted therapies for patients with $\mathrm{AH}$. The current evidence indicates that liver damage in $\mathrm{AH}$ is due to the complex interplay between ethanol metabolism, inflammation and innate immunity. Bird et al. (6) showed that both immunologic and nonimmunologic factors (such as cytokine injury and oxidative stress) play important roles in the pathogenesis of $\mathrm{AH}$. Chemotactic factors such as interleukin-8 (IL-8) cause migration of polymorphonuclear leukocytes to hepatic lobules in AH. In addition, tumor necrosis factor $\alpha(\mathrm{TNF} \alpha)$ has been sug- gested to play a pivotal role in AH and TNF superfamily receptors have been proved to be potential therapeutic targets in AH. Moreover, evidence showed that mitochondrial dysfunction acts as a common pathogenetic mechanism in several acute and chronic liver diseases including alcoholic and non-alcoholic fatty liver disease and hepatocellular carcinoma (7). In the pathological view of AH, a liver damage is similar to non-alcoholic fatty liver disease (NAFLD) (8). Oxidative stress is responsible for the progression from alcohol-induced fatty liver to $\mathrm{AH}$ and cirrhosis. Besides, evidence showed an interaction between $\mathrm{AH}$ and hepatocellular carcinoma. Alcoholic liver disease can lead to cirrhosis and there is underlying cirrhosis in many $\mathrm{AH}$ cases $(2,9)$. Additionally, hepatocellular carcinoma develops based on advanced liver cirrhosis and accounts for about 320000 deaths annually (10). Collectively, the pathogenesis of AH is multifactorial and more attention should be paid.

\section{Objectives}

In this study, to gain better insight into $\mathrm{AH}$, we screened differentially expressed genes (DEGs) in $\mathrm{AH}$ and con-

Copyright (C) 2015, Kowsar Corp. This is an open-access article distributed under the terms of the Creative Commons Attribution-NonCommercial 4.0 International License (http://creativecommons.org/licenses/by-nc/4.0/) which permits copy and redistribute the material just in noncommercial usages, provided the original work is properly cited. 
Liu M et al.

structed a protein-protein interaction (PPI) network of DEGs to further explore the molecular mechanism of AH.

\section{Materials and Methods}

\subsection{Data Sources}

The gene expression profile of GSE28619 was obtained from the National Center for Biotechnology Information (NCBI) Gene Expression Omnibus (GEO) database (http://www.ncbi.nlm.nih.gov/geo), which is based on the GPL570 [HG-U133_Plus_2] Affymetrix Human Genome U133 Plus 2.0 Array. Total 22 chips including 15 liver biopsies of AH group and 7 samples of normal livers (control group) were evaluated in this dataset. Liver biopsies in AH group were obtained using a transjugular approach and fragments of normal livers (control group) were obtained from optimal cadaveric liver donors $(n=3)$ or resection of liver metastases $(n=4)(11)$. An informed consent was obtained from each patient included and all the study protocols conformed to the ethical guidelines of the 1975 Declaration of Helsinki.

\subsection{Data Preprocessing and DEGs Screening}

We finally received the gene expression matrix by preprocessing the raw data as implemented in the Bioconductor AFFY package (12). Data was preprocessed through Robust Multichip Averaging (RMA) algorithm including background correction, normalization and probe summarization (13). We then used the two-tailed Student's ttests to identify the DEGs between $\mathrm{AH}$ and control groups $(\mathrm{P}<0.05, \mid \log 2$ fold change $(\mathrm{FC}) \mid>1)$.

\subsection{Gene Functional Annotation}

The functional annotation of DEGs was conducted to identify the transcription factors (TFs) based on the TRANSFAC database (http://transfac.gbf.de/TRANSFAC/). In addition, oncogenes and tumor suppressor genes were identified based on tumor suppressor genes (TSG) (14) and tumor associated genes (TAG) (15) database.

\subsection{Pathway and Functional Enrichment Analyses}

Kyoto encyclopedia of genes and genomes (KEGG) pathway (16) and gene ontology (GO) (17) functional analyses were performed to identify significantly enriched pathways and the biological processes of DEGs, respectively, using the online tool of the database for annotation, visualization and integrated discovery (DAVID, http://david. abcc.ncifcrf.gov/) (18) with P value $<0.01$. GO terms were identified under categories of biological process.

\subsection{PPI Network Construction and Subnetwork Mining}

DEGs were submitted to search tool for the retrieval of interacting genes (STRING) 9.1 (19). All interactions in
STRING were provided with a probabilistic confidence score (combined score), and in our analysis only interactions with combined score $>0.4$ were retained. PPI network was constructed by STRING and visualized using Cytoscape. The proteins in the network served as nodes and the degree of a node corresponded to the number of interactions with other proteins. The proteins with high degrees were considered as the hub nodes. In addition, we further performed subnetwork mining in the PPI network based on BioNet with FDR less than 0.00000005 . The subnetwork was visualized in Cytoscape.

\section{Results}

\subsection{DEGs Between AH and Control Groups}

A total of 908 DEGs were identified, including 551 upregulated genes and 357 down-regulated genes. Among these DEGs, there were four up-regulated TFs (SOX9, SOX4, NME2 and ENO1) and 21 down-regulated TFs (such as MME, JUNB, JUN, FOS and ZFP36), as well as 57 up-regulated TAGs (10 oncogenes, 37 tumor suppressor genes and 10 other genes, which were uncertain on tumor development) and 28 down-regulated TAGs ( 4 oncogenes, 21 tumor suppressor genes and 3 other genes) (Table 1). Parts of the up-regulated oncogenes were RBM3, PTTG1, PDGFRA, LCN2 and $L A M C 2$; parts of the up-regulated tumor suppressor genes were VWA5A, UCHL1, TUSC3, TPM1 and NME1; parts of the up-regulated other genes were SRPX2, SQSTM1, S100A11, $M G P$ and GLS. The down-regulated oncogenes were MME, JUNB, JUN and FOS; the down-regulated tumor suppressor genes included ZFP36, TGFBR3, SMARCA2, SIK1 and PHLPP1; the down-regulated other genes were RHOB, NR4A2 and MCC.

Table 1. The DEGs Between AH and Control Groups ${ }^{a}$

\begin{tabular}{lccc}
\hline Variables & $\begin{array}{c}\text { Total DEGs } \\
\text { Counts }\end{array}$ & TFs Counts TAG Counts \\
\hline Up-regulated & 551 & 4 & 57 \\
Down-regulated & 357 & 21 & 28 \\
Total & 908 & 25 & 85 \\
\hline
\end{tabular}

a Abbreviations: DEGs, differentially expressed genes; Total DEGs counts, number of total DEGs; TFs Counts, number of transcription factors (TFs); TAGs Counts, number of Tumor Associated Genes (TAGs).

\subsection{KEGG Pathway Enrichment Analysis of Up-and Down-Regulated DEGs}

The up-regulated DEGs were enriched in 15 pathways, which mainly involved in pathways related to cancer such as extracellular matrix (ECM)-receptor interaction, focal adhesion, cytokine-cytokine receptor interaction pathways and pathways in cancer. Meanwhile, the downregulated DEGs were enriched in 22 pathways, which were mainly about metabolic pathways. The top five significantly enriched pathways of up and down regulated DEGs are listed in Table 2. 
Liu M et al.

\subsection{GO Term (Biological Processes) Enrichment Analysis of up- and Down-Regulated DEGs}

The up-regulated DEGs were significantly enriched in 112 biological processes and the top five significantly enriched processes were mainly related to ECM and cell adhesion (Table 3). Meanwhile, the down-regulated DEGs were significantly enriched in 84 biological processes and the top five significantly enriched processes mainly involved in material metabolisms (Table 3).

\begin{tabular}{lcc}
\hline Table 2. Top Five Significantly Enriched Pathways for DEGs ${ }^{\mathrm{a}}$ \\
\hline KEGG Pathway & Gene Counts & P Value \\
\hline Up-regulated & & \\
\hline ECM-receptor interaction & 21 & $2.07 \mathrm{E}-12$ \\
\hline Focal adhesion & 13 & $8.27 \mathrm{E}-10$ \\
\hline Protein digestion and absorption & 13 & $2.53 \mathrm{E}-06$ \\
\hline Gap junction & 14 & $3.35 \mathrm{E}-05$ \\
\hline Amoebiasis & & \\
Down-regulated & 67 & $8.12 \mathrm{E}-13$ \\
\hline Metabolic pathways & 8 & $9.92 \mathrm{E}-07$ \\
\hline $\begin{array}{l}\text { Glycine, serine and threonine } \\
\text { metabolism }\end{array}$ & 5 & 0.000195 \\
\hline Circadian rhythm-mammal & 8 & 0.000208 \\
\hline Retinol metabolism & 8 & 0.000429 \\
\hline Bile secretion & 5 & \\
\hline
\end{tabular}

a Abbreviations: DEGs, differentially expressed genes; ECM, extracellular matrix; Gene Counts, number of genes; KEGG, Kyoto Encyclopedia of Genes and Genomes.

Table 3. Top Five Significantly Enriched Biology Processes for DEGs ${ }^{\text {a }}$

\begin{tabular}{|cccc}
\hline GO ID & Term & $\begin{array}{c}\text { Gene } \\
\text { Counts }\end{array}$ & P Value \\
\hline Up-regulated & extracellular matrix orga- \\
G0:0030198 & 50 & 0 \\
\hline G0:0031012 & $\begin{array}{c}\text { extracellular matrix } \\
\text { extracellular space }\end{array}$ & 49 & $4.44 \mathrm{E}-16$ \\
\hline G0:0005615 & cell adhesion & 72 & 1.15E-11 \\
\hline GO:0007155 & $\begin{array}{c}\text { extracellular membrane- } \\
\text { bounded organelle }\end{array}$ & 17 & $9.39 \mathrm{E}-11$ \\
\hline GO:0065010 & & & \\
\hline
\end{tabular}

\section{Down-regu-}

lated

\begin{tabular}{|c|ccc|}
\hline GO:0044281 & $\begin{array}{c}\text { small molecule metabolic } \\
\text { process }\end{array}$ & 117 & 0 \\
\hline GO:0009063 & $\begin{array}{c}\text { cellular amino acid cata- } \\
\text { bolic process }\end{array}$ & 18 & $8.72 \mathrm{E}-12$ \\
\hline GO:0010035 & $\begin{array}{c}\text { response to inorganic } \\
\text { substance }\end{array}$ & 26 & $1.83 \mathrm{E}-09$ \\
\hline GO:0008202 & steroid metabolic process & 22 & $3.55 \mathrm{E}-08$ \\
\hline GO:0006006 & glucose metabolic process & 19 & $8.00 \mathrm{E}-08$ \\
\hline
\end{tabular}

a Abbreviations: DEGs, differentially expressed genes; GO, Gene Ontology; Gene Counts, number of genes.

\section{Figure 1. PPI Network in $\mathrm{AH}$}

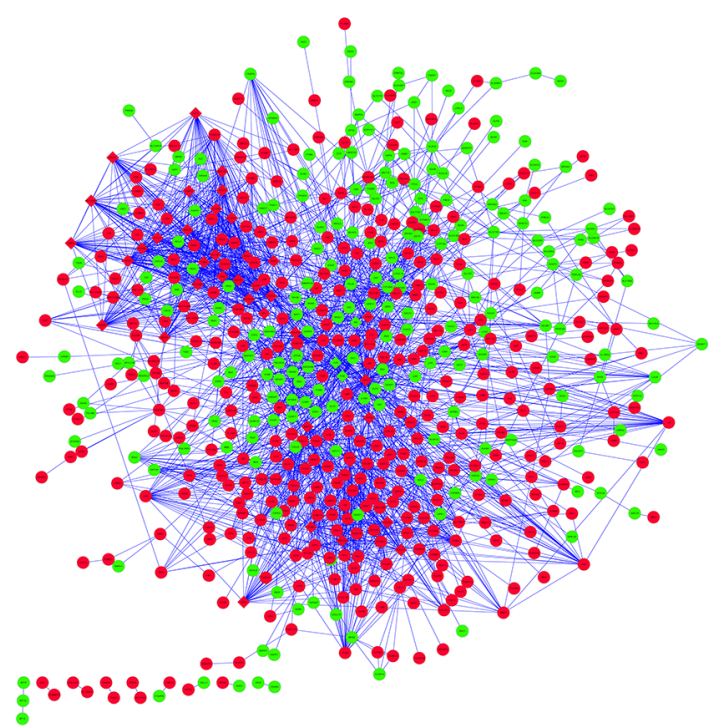

Red nodes represent up-regulated DEGs; green nodes represent downregulated DEGs; rhombic nodes represent key nodes; Blue lines stand for the interaction between two proteins.

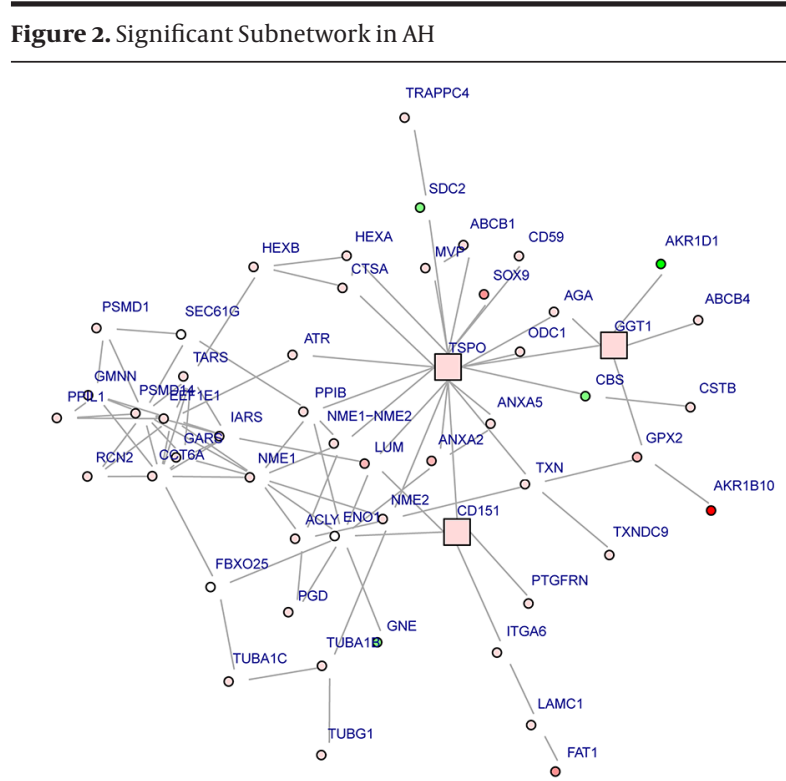

Red nodes represent up-regulated DEGs; green nodes represent down-regulated DEGs; square nodes represent negative-scoring genes additionally included in the optimal solution. The depth of color of nodes is related to the fold change of the DEGs.

\subsection{PPI Network and Subnetwork of DEGs}

The PPI network including 608 nodes and 2878 interactions was constructed (Figure 1). The connectivity degree of each node in this PPI network was calculated and the 
Liu M et al.

top five nodes with degrees $\geq 58$ were TSPO (translocator protein), JUN (transcription factor AP-1), PCNA (proliferating cell nuclear antigen), FOS (proto-oncogene c-Fos) and CDK1 (cyclin-dependent kinase 1). Furthermore, one subnetwork including 53 nodes and 131 interactions was constructed (Figure 2). TSPO with the highest degree (20) in the subnetwork was considered as the hub node, which interacted with many proteins including PPIB (peptidylprolyl cis-trans isomerase B), NME1 (nucleoside diphosphate kinase A) and NME2 (nucleoside diphosphate kinase B).

\section{Discussion}

According to the gene expression profile analysis between $\mathrm{AH}$ and control groups, 551 up-regulated and 357 down-regulated DEGs were obtained. We next constructed the PPI network between the DEGs and extracted one subnetwork. In the subnetwork, TSPO was the node with highest degree and found to interact with 20 proteins such as PPIB, NME1 and NME2. We found that TSPO (encoding a translocator protein, $18 \mathrm{kDa}$ ), an up-regulated gene in $\mathrm{AH}$, was enriched in some biological processes mainly including regulation of reactive oxygen species metabolic process, response to alcohol, negative regulation of nitric oxide biosynthetic process and regulation of necrotic cell death. TSPO is a nucleus-encoded mitochondrial target trans-membrane protein (also known as peripheral-type benzodiazepine receptor, PBR) able to modulate the function of mitochondria $(20,21)$. Evidence showed that mitochondrial dysfunction is known to be a contributing factor to chronic alcohol induced liver injury (22). Ethanol-elicited alterations in mitochondria structure and function have been demonstrated to induce oxidative stress in liver $(23,24)$. In addition, reactive oxygen species (ROS) and reactive nitrogen species (RNS) have been identified as key components in the progression from alcohol-induced fatty liver to $\mathrm{AH}$ and cirrhosis $(25,26)$. Moreover, Xie et al. (27) indicated that TSPO correlates with mitochondrial dysfunction, which is responsible for liver damage and disease progression in NAFLD. Therefore, we considered that the oxidative stress of liver induced by mitochondrial dysfunction might be responsible for liver damage and disease progression in AH, as well TSPO may play important role in it. The PPI subnetwork in our study showed that TSPO directly interacted with PPIB (also named cyclophilin B). PPIB can bind to cells derived from T- lymphocytes and B- lymphocytes and may regulate cyclosporine Amediated immunosuppression (28). Alcohol consumption can increase the translocation of bacteria-derived lipopolysaccharide (LPS) from the gut to the liver as well as activate innate immunity components. Hence, PPIB might play crucial role in innate immunity in $\mathrm{AH}$. In addition, some studies supported that PPIB has an interaction with CD147 (a glycoprotein, a receptor for PPIB) (29). CD147 is important for mitochondrial respiration and can promote activation of hepatic stellate cells and be a target for antibody therapy of liver fibrosis $(29,30)$. In addition, TSPO has been found in colocalization with the mitochondrial manganese-dependent superoxide dismutase (a ROS scavenger) in the liver $(31,32)$. ROS is produced during the activation of innate immunity (33). Considering that TSPO could modulate the function of mitochondria $(20,21)$ and had effects on the immune, we speculated that the interaction between TSPO and PPIB might play important role in liver damage in $\mathrm{AH}$ and responsible for the progression of fibrosis from $\mathrm{AH}$. In addition, the PPI subnetwork in our study showed that NME1 (also named Nm 23) directly interacted with NME2 (also named Nm 23B). Human NME1 and NME2 have 88\% homology and NME2 was isolated based on sequence homology to NM23-H1 $(34,35)$. Ohneda et al. (36) suggested that NME1 and NME2 have a role in the initial stages of tumorigenesis. Besides, studies showed that NME1 is the first of 13 identified tumor metastasis suppressor genes $(37,38)$. The reducing protein and mRNA expressions of NME1 in tumor samples are correlated with characteristics of aggressive cancer, such as poor clinical prognosis and survival, lymph node infiltration, as well as invasiveness and metastasis in a variety of tumor types, including hepatocellular carcinoma $(39,40)$. The study by Yamaguchi et al. (41) showed that NME2 mRNA is abundantly expressed in hepatocellular carcinoma tissues and established hepatoma cell lines. Moreover, NME1 may play a more important role than NME2 in intrahepatic metastasis in hepatocellular carcinoma (40). A previous study indicated that alcohol abuse could increase the risk of hepatocellular carcinoma (42). Liver damage ranged from acute hepatitis to hepatocellular carcinoma is mainly through apoptosis, necrosis, inflammation, immune response and fibrosis, all processes that involve hepatocyte (42). In our study, NME1 as an up-regulated tumor suppressor gene, was enriched in some biological processes in $\mathrm{AH}$, mainly including regulation of response to alcohol, response to steroid hormone stimulus and response to ketone. NME2as an up-regulated DEG was enriched in some biological processes in $\mathrm{AH}$ including cell adhesion, ruffle and cytosol. Therefore, we supposed that the interaction between NME1 and NME2 might play key roles in the development of $\mathrm{AH}$ transformed hepatocellular carcinoma. In conclusion, we analyzed the gene expression profile of $\mathrm{AH}$ using bioinformatics analysis and found that TSPO might contribute to the liver damage and $\mathrm{AH}$ progression induced by mitochondrial dysfunction through oxidative stress of liver. TSPO could interact with PPIB and their interaction might play an important role in liver damage in $\mathrm{AH}$ and responsible for the progression of fibrosis from $\mathrm{AH}$. Additionally, the interaction between NME1 and NME2 might paly crucial roles in development of hepatocellular carcinoma in patients with AH. However, specific roles and mechanisms of these DEGs in AH should be investigated and confirmed in further in vivo and vitro studies. 
Liu M et al.

\section{Authors' Contributions}

Minghui Liu participated in study design. Yuchang Dou performed the statistical analysis. Ran Sun and Yonggui Zhang performed the study, collected important background information and drafted the manuscript. Yansong Liu conceived of this study and participated in design and helped to draft the manuscript. All authors read and approved the final manuscript.

\section{References}

1. Nagata K, Suzuki H, Sakaguchi S. Common pathogenic mechanism in development progression of liver injury caused by non-alcoholic or alcoholic steatohepatitis. J Toxicol Sci. 2007;32(5):453-68.

2. Lucey MR, Mathurin P, Morgan TR. Alcoholic hepatitis. N Engl J Med. 2009;360(26):2758-69.

3. Spengler EK, Dunkelberg J, Schey R. Alcoholic hepatitis: current management. Dig Dis Sci. 2014;59(10):2357-66.

4. Singal AK, Walia I, Singal A, Soloway RD. Corticosteroids and pentoxifylline for the treatment of alcoholic hepatitis: Current status. World J Hepatol. 2011;3(8):205-10.

5. Chayanupatkul M, Liangpunsakul S. Alcoholic hepatitis: a comprehensive review of pathogenesis and treatment. World J Gastroenterol. 2014;20(20):6279-86.

6. Bird G. Interleukin-8 in alcoholic liver disease. Acta Gastroenterol Belg. 1994;57(3-4):255-9.

7. Serviddio G, Bellanti F, Sastre J, Vendemiale G, Altomare E. Targeting mitochondria: a new promising approach for the treatment of liver diseases. Curr Med Chem. 2010;17(22):2325-37.

8. Abangah G, Yousefi A, Asadollahi R, Veisani Y, Rahimifar P, Alizadeh S. Correlation of Body Mass Index and Serum Parameters With Ultrasonographic Grade of Fatty Change in Non-alcoholic Fatty Liver Disease. Iran Red Crescent Med J. 2014;16(1).

9. O'Shea RS, Dasarathy S, McCullough AJ, Practice Guideline Committee of the American Association for the Study of Liver D, Practice Parameters Committee of the American College of G. Alcoholic liver disease. Hepatology. 2010;51(1):307-28.

10. Alavian SM. Networking for Overcoming on Viral Hepatitis in Middle East and Central Asia:" Asian Hepatitis Network. Hepat Mon. 2007;7(4):181-2.

11. Affo S, Dominguez M, Lozano JJ, Sancho-Bru P, Rodrigo-Torres D, Morales-Ibanez O, et al. Transcriptome analysis identifies TNF superfamily receptors as potential therapeutic targets in alcoholic hepatitis. Gut. 2013;62(3):452-60.

12. Gautier L, Cope L, Bolstad BM, Irizarry RA. affy--analysis of Affymetrix GeneChip data at the probe level. Bioinformatics. 2004;20(3):307-15.

13. Irizarry RA, Hobbs B, Collin F, Beazer-Barclay YD, Antonellis KJ, Scherf U, et al. Exploration, normalization, and summaries of high density oligonucleotide array probe level data. Biostatistics. 2003;4(2):249-64.

14. Zhao M, Sun J, Zhao Z. TSGene: a web resource for tumor suppressor genes. Nucleic Acids Res. 2013;41(Database issue):D970-6.

15. Chen JS, Hung WS, Chan HH, Tsai SJ, Sun HS. In silico identification of oncogenic potential of fyn-related kinase in hepatocellular carcinoma. Bioinformatics. 2013;29(4):420-7.

16. Arakawa K, Kono N, Yamada Y, Mori H, Tomita M. KEGG-based pathway visualization tool for complex omics data. In Silico Biol. 2005;5(4):419-23.

17. Hulsegge I, Kommadath A, Smits MA. Globaltest and GOEAST: two different approaches for Gene Ontology analysis. BMC Proc. 2009;3(Suppl 4):S10.

18. Huang da W, Sherman BT, Lempicki RA. Systematic and integrative analysis of large gene lists using DAVID bioinformatics resources. Nat Protoc. 2009;4(1):44-57.

19. Franceschini A, Szklarczyk D, Frankild S, Kuhn M, Simonovic M, Roth A, et al. STRING v9.1: protein-protein interaction networks, with increased coverage and integration. Nucleic Acids Res. 2013;41(Database issue):D808-15.
20. Papadopoulos V, Baraldi M, Guilarte TR, Knudsen TB, Lacapere JJ, Lindemann P, et al. Translocator protein (18kDa): new nomenclature for the peripheral-type benzodiazepine receptor based on its structure and molecular function. Trends Pharmacol Sci. 2006;27(8):402-9.

21. Casellas P, Galiegue S, Basile AS. Peripheral benzodiazepine receptors and mitochondrial function. Neurochem Int. 2002;40(6):47586.

22. Mantena SK, King AL, Andringa KK, Landar A, Darley-Usmar V, Bailey SM. Novel interactions of mitochondria and reactive oxygen/ nitrogen species in alcohol mediated liver disease. World J Gastroenterol. 2007;13(37):4967-73.

23. Bailey SM. A review of the role of reactive oxygen and nitrogen species in alcohol-induced mitochondrial dysfunction. Free Radic Res. 2003;37(6):585-96.

24. Andringa KK, King AL, Eccleston HB, Mantena SK, Landar A, Jhala $\mathrm{NC}$, et al. Analysis of the liver mitochondrial proteome in response to ethanol and S-adenosylmethionine treatments: novel molecular targets of disease and hepatoprotection. Am J Physiol Gastrointest Liver Physiol. 2010;298(5):G732-45.

25. Loguercio C, Federico A. Oxidative stress in viral and alcoholic hepatitis. Free Radic Biol Med. 2003;34(1):1-10

26. Wu D, Cederbaum AI. Oxidative stress and alcoholic liver disease. Semin Liver Dis. 2009;29(2):141-54

27. Xie L, Yui J, Hatori A, Yamasaki T, Kumata K, Wakizaka H, et al Translocator protein $(18 \mathrm{kDa})$, a potential molecular imaging biomarker for non-invasively distinguishing non-alcoholic fatty liver disease. J Hepatol. 2012;57(5):1076-82.

28. Price ER, Zydowsky LD, Jin MJ, Baker CH, McKeon FD, Walsh CT Human cyclophilin B: a second cyclophilin gene encodes a peptidyl-prolyl isomerase with a signal sequence. Proc Natl Acad Sci U S A. 1991;88(5):1903-7.

29. Yurchenko V, O'Connor M, Dai WW, Guo H, Toole B, Sherry B, et al CD147 is a signaling receptor for cyclophilin B. Biochem Biophys Res Commun. 2001;288(4):786-8.

30. Zhang DW, Zhao YX, Wei D, Li YL, Zhang Y, Wu J, et al. HAb18G CD147 promotes activation of hepatic stellate cells and is a target for antibody therapy of liver fibrosis. J Hepatol. 2012;57(6):1283-91.

31. Forman HJ, Fukuto JM, Torres M. Redox signaling: thiol chemistry defines which reactive oxygen and nitrogen species can act as second messengers. Am J Physiol Cell Physiol. 2004;287(2):C24656.

32. Kohchi C, Inagawa H, Nishizawa T, Soma G. ROS and innate immunity. Anticancer Res. 2009;29(3):817-21.

33. Fischer R, Schmitt M, Bode JG, Haussinger D. Expression of the peripheral-type benzodiazepine receptor and apoptosis induction in hepatic stellate cells. Gastroenterology. 2001;120(5):1212-26.

34. Stahl JA, Leone A, Rosengard AM, Porter L, King CR, Steeg PS. Identification of a second human $\mathrm{nm} 23$ gene, nm23-H2. Cancer Res. 1991;51(1):445-9.

35. Lee MJ, Xu DY, Li H, Yu GR, Leem SH, Chu IS, et al. Pro-oncogenic potential of NM23-H2 in hepatocellular carcinoma. Exp Mol Med. 2012;44(3):214-24.

36. Ohneda K, Fukuda M, Shimada N, Ishikawa N, Ichou T, Kaji K, et al Increased expression of nucleoside diphosphate kinases/nm23 in human diploid fibroblasts transformed by SV40 large T antigen or 60Co irradiation. FEBS Lett. 1994;348(3):273-7.

37. Steeg PS, Bevilacqua G, Kopper L, Thorgeirsson UP, Talmadge JE, Liotta LA, et al. Evidence for a novel gene associated with low tumor metastatic potential.J Natl Cancer Inst. 1988;80(3):200-4.

38. Palmieri D, Horak CE, Lee JH, Halverson DO, Steeg PS. Translational approaches using metastasis suppressor genes.J Bioenerg Biomembr. 2006;38(3-4):151-61.

39. Steeg PS. Tumor metastasis: mechanistic insights and clinical challenges. Nat Med. 2006;12(8):895-904.

40. Iizuka N, Oka M, Noma T, Nakazawa A, Hirose K, Suzuki T. NM23$\mathrm{H} 1$ and NM23-H2 messenger RNA abundance in human hepatocellular carcinoma. Cancer Res.1995;55(3):652-7.

41. Yamaguchi A, Urano T, Goi T, Takeuchi K, Niimoto S, Nakagawara $\mathrm{G}$, et al. Expression of human $\mathrm{nm} 23-\mathrm{H} 1$ and $\mathrm{nm} 23-\mathrm{H} 2$ proteins in hepatocellular carcinoma. Cancer.1994;73(9):2280-4.

42. Morgan TR, Mandayam S, Jamal MM. Alcohol and hepatocellular carcinoma. Gastroenterology. 2004;127(5 Suppl 1):S87-96. 